\title{
Communication
}

\section{Filamentary High-Resolution Electrical Probes for Nanoengineering}

Eugene Soh, Ghazi Syed, Giulio Mazzotta, Benjamin F. Porter, Moritz K. Riede, Robin Nicholas, Judy S. Kim, and Harish Bhaskaran

Nano Lett., Just Accepted Manuscript • DOI: 10.1021/acs.nanolett.9b04302 • Publication Date (Web): 06 Jan 2020

Downloaded from pubs.acs.org on January 13, 2020

\section{Just Accepted}

"Just Accepted" manuscripts have been peer-reviewed and accepted for publication. They are posted online prior to technical editing, formatting for publication and author proofing. The American Chemical Society provides "Just Accepted" as a service to the research community to expedite the dissemination of scientific material as soon as possible after acceptance. "Just Accepted" manuscripts appear in full in PDF format accompanied by an HTML abstract. "Just Accepted" manuscripts have been fully peer reviewed, but should not be considered the official version of record. They are citable by the Digital Object Identifier (DOI®). "Just Accepted" is an optional service offered to authors. Therefore, the "Just Accepted" Web site may not include all articles that will be published in the journal. After a manuscript is technically edited and formatted, it will be removed from the "Just Accepted" Web site and published as an ASAP article. Note that technical editing may introduce minor changes to the manuscript text and/or graphics which could affect content, and all legal disclaimers and ethical guidelines that apply to the journal pertain. ACS cannot be held responsible for errors or consequences arising from the use of information contained in these "Just Accepted" manuscripts. 
Filamentary High-Resolution Electrical Probes for Nanoengineering

Eugene J.H. Soh, $\uparrow$ Syed Ghazi Sarwat, $\uparrow \#$ Giulio Mazzotta, $\nRightarrow$ Benjamin F. Porter, $†$ Moritz Riede, $\neq$ Robin Nicholas, $\ddagger$ Judy S. Kim, $†$ and Harish Bhaskaran*†

†Department of Materials, University of Oxford, Oxford OX13PH, United Kingdom

‡Clarendon Laboratory, Department of Physics, University of Oxford, OX1 3PU, United Kingdom

\#Present: IBM Research - Zurich, Rüschlikon, 8803, Switzerland

E-mail: harish.bhaskaran@materials.ox.ac.uk

Phone: +44 1865273772

\section{Abstract}

Confining electric fields to a nanoscale region is challenging yet crucial for applications such as high resolution probing of electrical properties of materials and electric-field manipulation of nanoparticles. State-of-the-art techniques involving atomic force microscopy typically have a lateral resolution limit of tens of nanometers due to limitations in the probe geometry and stray electric fields that extend over space. Engineering the probes is the most direct approach to improving this resolution limit. However, current methods to fabricate highresolution probes, which can effectively confine the electric fields laterally involve expensive and sophisticated probe manipulation, which has limited the use of this approach. Here, we demonstrate that nanoscale phase switching of configurable thin films on probes can result in high-resolution electrical probes. These configurable coatings can be both germanium-antimony-tellurium (GST) as well as amorphous-carbon, materials known to undergo electric field-induced non-volatile, yet reversible switching. By forming a localized conductive filament 


\begin{abstract}
through phase transition, we demonstrate a spatial resolution of electrical field beyond the geometrical limitations of commercial platinum probes (i.e. an improvement of $\sim 48 \%$ ). We then utilize these confined electric fields to manipulate nanoparticles with single nanoparticle precision via dielectrophoresis. Our results advance the field of nanomanufacturing and metrology with direct applications for pick and place assembly at the nanoscale.
\end{abstract}

Keywords: Atomic Force Microscopy, Kelvin Probe Force Microscopy, Resistive Switching, High Resolution, Dielectrophoresis

\title{
Introduction
}

The ability to effectively probe and modulate the electrical properties at the nanoscale is vital to both building higher-performing devices and in understanding the material properties, for applications that span from transistors to catalysis to energy harvesting. One most commonly used tool to characterize properties at the nanoscale is the atomic force microscope (AFM) ${ }^{1-12}$. Although AFM can achieve angstrom-scale resolution in the vertical direction, its lateral resolution is inherently bound by the tip geometry, which in commercial probes, tends to be on the order of tens of nanometers. Lateral resolution is even more challenging when probing electronic properties at the nanoscale using conductive coatings, such as Kelvin probe force microscopy (KPFM) and current-voltage characteristics using conductive AFM. Specialised probes have been developed to overcome this limitation of lower lateral resolution. For example, encapsulated tips with a nanoscale conducting core have been developed to improve electrical isolation and reduce tip wear ${ }^{13}$. Cantilevers have also been specially engineered with higher mechanical resonance modes so as to improve resolution of the KPFM measurements ${ }^{14}$. Additionally, tips can be modified with insulator shielding ${ }^{15}$ or with carbon nanotubes ${ }^{16-}$ 18 to improve lateral resolution. However, specialized experimental techniques are required to employ such probes, which by themselves are difficult to fabricate, all of which 
have limited the application of such techniques ${ }^{19}$.

In this paper, we demonstrate a new type of electrical probe tip based on electrically reconfigurable coatings with high lateral resolution. We present two applications of our high-resolution probes: (a) enhancing the existing geometrical resolution limits and (b) capturing single gold nanoparticles. Both germanium-antimony-tellurium (GST) and amorphous carbon were used as the electrically reconfigurable coating. The electrical resistance of GST exhibits a decrease by over two orders of magnitude when switched from an amorphous to crystalline state in an AFM ${ }^{20}$. Similarly, amorphous carbon has been shown to exhibit field-induced threshold switching at the nanoscale, from a high resistance state (HRS) to a low resistance state (LRS) in the presence of high electric fields due to clustering of $\mathrm{sp}^{2}$ sites within the $\mathrm{sp}^{3}$ matrix ${ }^{21}$. Taking advantage of this property, we coat an AFM probe tip with either GST or amorphous carbon and induce switching locally at the tip apex. Figure 1 illustrates how creating a conductive filament can result in an electrical probe that is much smaller in dimension as compared to the tip radius. This allows enhanced resolution of electrical measurements due to confinement of the electric field. We demonstrate this by carrying out KPFM on carbon nanotubes of about $10 \mathrm{~nm}$ in diameter and by picking up $20 \mathrm{~nm}$ gold nanoparticles on the tip apex.

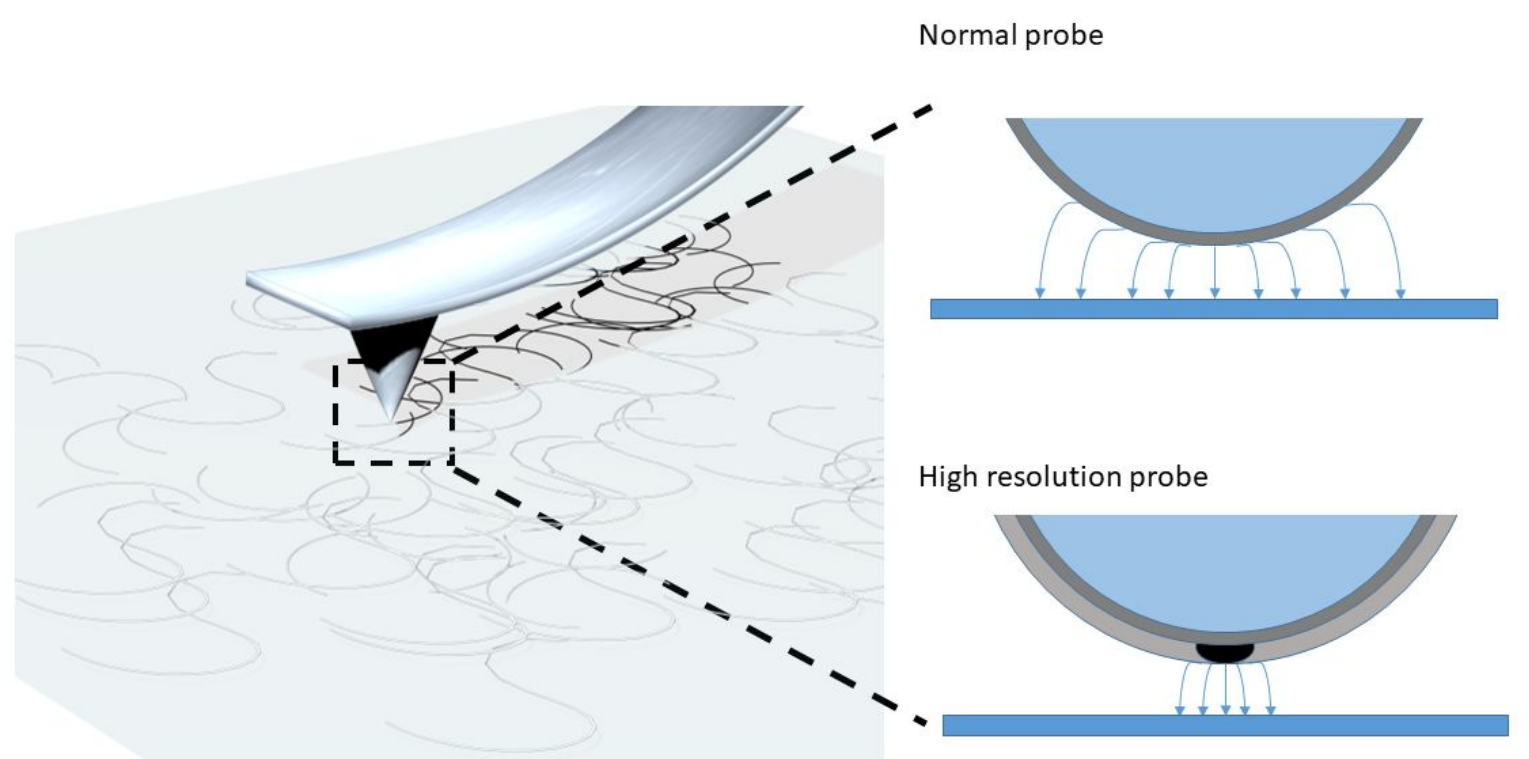

Figure 1: Schematic of an AFM tip illustrating how a conductive filament can enhance 
the resolution of electrical measurements. The high-resolution probe has a small volume of low resistance coating surrounded by the higher resistance coating. This confines the electric field to a region smaller than the dimensions of the probe tip, thus enhancing electrical resolution.

Creation of filamentary probes

After coating the tip with either amorphous carbon or GST, a conductive filament is created via conductive AFM (more details in the Methods section). To determine if phase switching at the tip apex has occurred, the IV curve is monitored as the voltage between the tip and substrate is ramped up. One such switching curve is shown in Fig. 2(a). The resistance of the tip-sample contact remains high until the threshold switching voltage is reached. The current measured spikes at about $3 \mathrm{~V}$. On the reverse voltage sweep, the current decreases linearly and the slope indicates a lower resistance compared to the slope during the forward voltage sweep. A subsequent low amplitude voltage sweep (i.e. $100 \mathrm{mV}$ ) is conducted to confirm that the tip-sample contact is now highly conductive. This confirms that the switching of amorphous to conductive state is non-volatile. This non-volatility is crucial in ensuring high lateral resolution during subsequent electrical AFM measurements. We find that once switched, the probes remain in LRS for several days (See Fig. S1). The switching process is similar for both carbon and GST coated tips. However, we believe that the mechanism behind electric field confinement is different. With GST, it is well known that there is a phase change between amorphous and crystalline state that changes the resistance of the material [21]. As for the mechanism in carbon, it could be due to carbon reconfiguration ${ }^{21}$ or the possibility of metal migration into the carbon which creates a metallic filamentation ${ }^{22}$.

We hypothesize that the switching from HRS to LRS of carbon in our experiments is due to reconfiguration of carbon rather than metallic filamentation. To test this hypothesis, we conducted the following four sets of experiments: (a) scaling behaviour 
due to thickness of carbon, (b) different probe materials, (c) different substrates and (d) switching with reverse voltage. We coated platinum AFM tips with different thickness of carbon and observed the switching voltage (Fig. 2(b)). It is observed that the switching voltage increases as coating thickness increases. However, this trend is not linear as was expected. This may be due to the thinness of coating used where any slight variations in coating thickness and accuracy in measuring the thickness can affect this result. Furthermore, we coated carbon onto AFM tips of different materials (i.e. platinum, silicon and diamond) and conducted threshold switching on highly oriented pyrolytic graphite (HOPG) (Fig. 2(c)). Silicon and diamond are materials not known to undergo any kind of reconfiguration or phase change. Threshold switching was also observed on platinum, indium tin oxide (ITO) and HOPG substrates (Fig. 2(d)), thus ruling out metallic filament formation. Furthermore, when the voltage bias is reversed, threshold switching is observed. Since metallic filamentation is polarity dependent, we can conclude that the change in HRS to LRS is due to reconfiguration of carbon. Additionally, no significant metal signal was found by EDX in the carbon coating (Fig. S5). The experiments described above suggest that reconfiguration of amorphous carbon is the mechanism behind the threshold switching behaviour and hence the creation of highresolution electrical probes. 

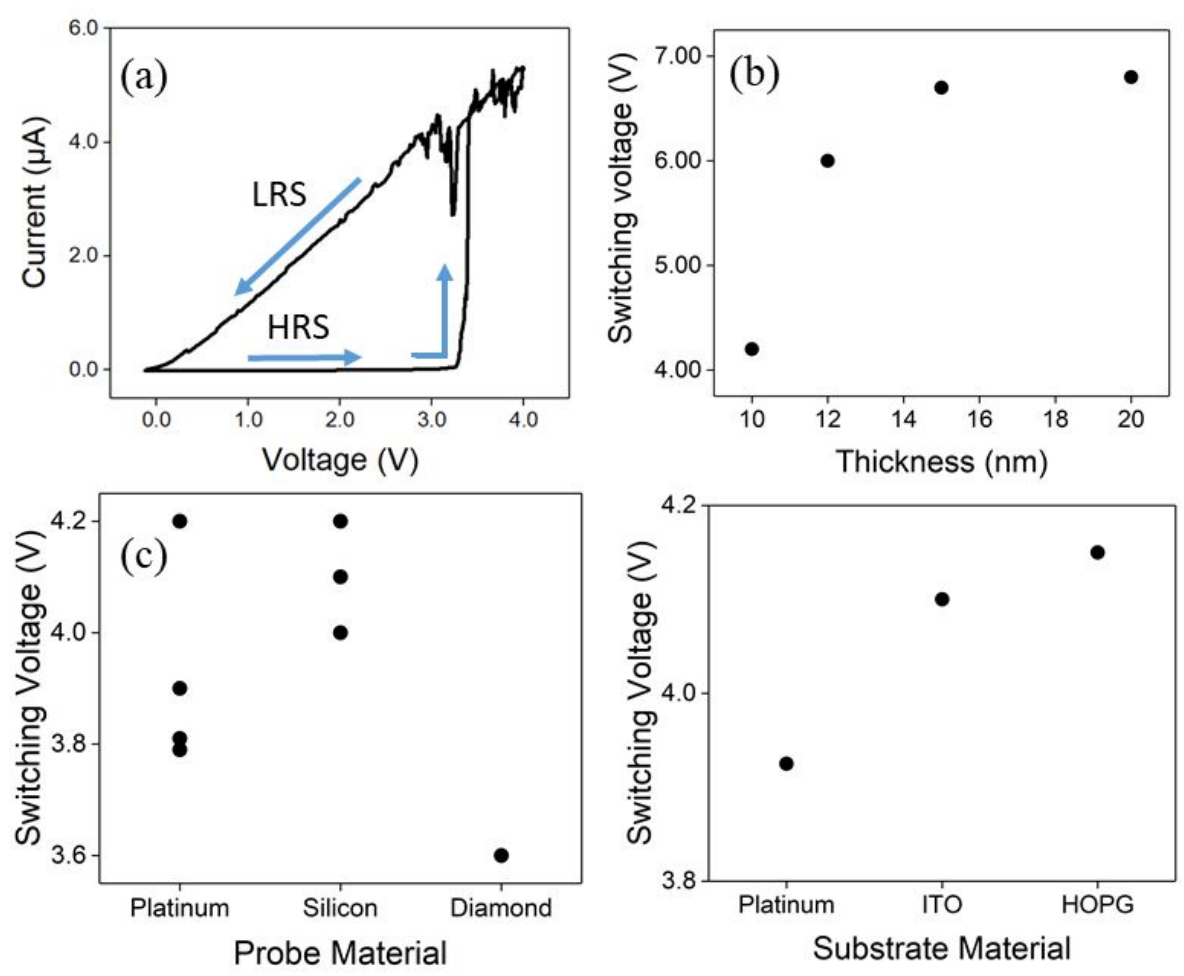

Figure 2: (a) Representative IV curve measured when switching the carbon coating phase on the AFM tip. As the voltage is ramped up from $0 \mathrm{~V}$ to $4 \mathrm{~V}$, the sudden spike in current at about $3 \mathrm{~V}$ suggests a change from a high resistance state to a low resistance state. A linear line on the reverse ramp indicates that the phase change is non-volatile. (b) Switching voltage vs. thickness of amorphous carbon coating. (c) Switching voltages of varied composition probes coated with $10 \mathrm{~nm}$ of amorphous carbon. (d) Switching voltages on different substrate materials using silicon probes coated with $10 \mathrm{~nm}$ of carbon.

Filamentary Probes for Kelvin Probe Force Microscopy

KPFM is a variant of non-contact mode AFM that measures the contact potential difference (CPD) between the tip and sample. In this way, the work function of the sample 
can be calculated and mapped out with nanometer-scale precision. KPFM is used in the study of organic materials and device ${ }^{23,24}$, heterostructures ${ }^{25}$ and biological materials 26,27. The basic principle of KPFM involves applying an alternating current voltage to the cantilever and tracking its response. The electrostatic force between the tip and sample is minimized by applying direct current, equivalent to the contact potential difference, to the tip. There are two methods to track the response of the cantilever: Amplitude modulation (AM) and frequency modulation (FM). KPFM is commonly carried out in AM mode due to ease of implementation. On the other hand, FM mode enables better resolution than AM mode due to improved tip-sample sensitivity. Here, we demonstrate how the use of two different phase change material coatings (i.e. GST and amorphous carbon) can produce high-resolution electrical probes in both AM- and FM-KPFM. These carbon-coated tips are advantageous over GST as they are widely available and can be readily coated onto commercially available tips. 

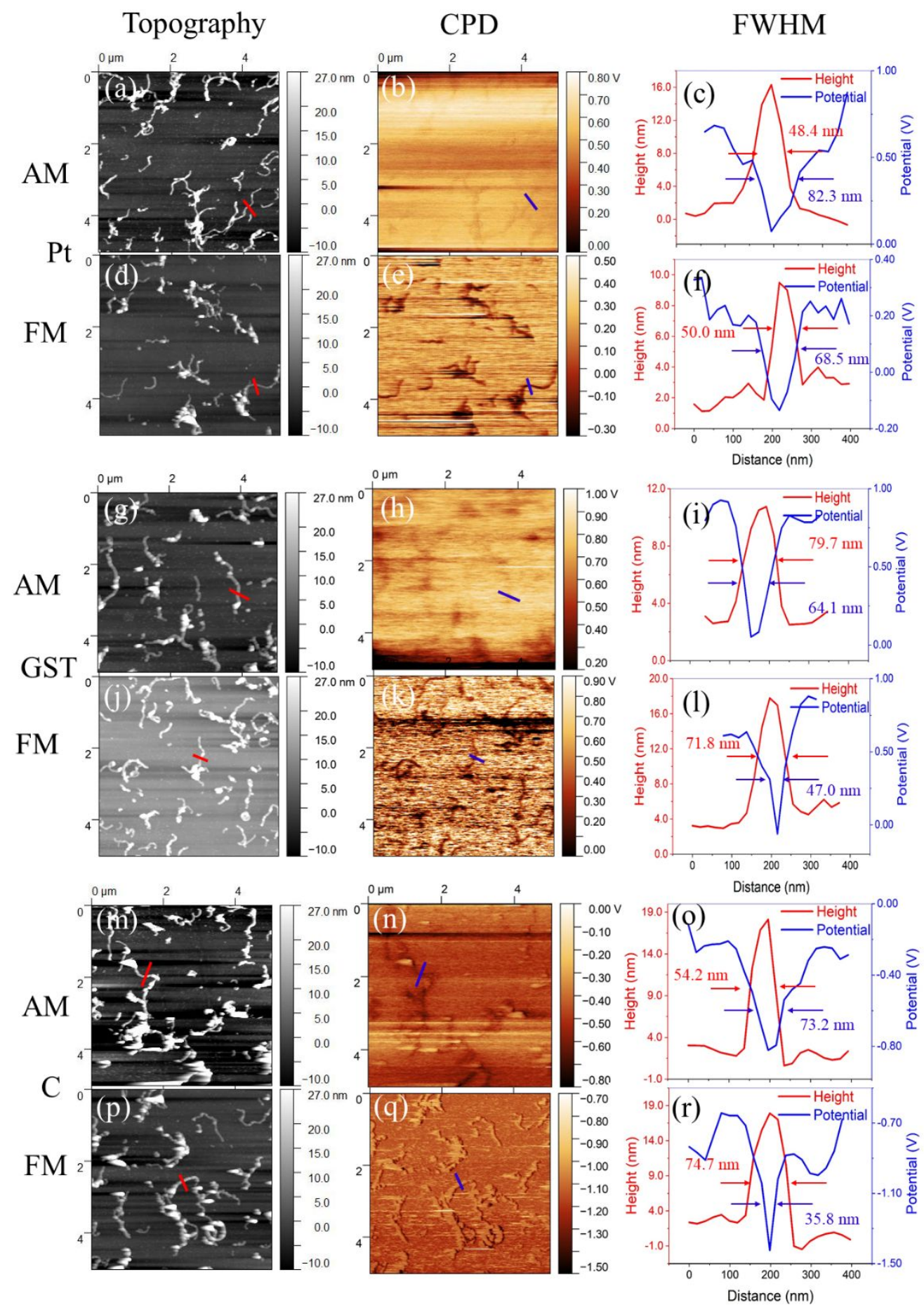

Figure 3: Topography, CPD, and the corresponding line sections of scans conducted with (a-f) platinum tips, (g-l) GST and (m-r) carbon tips with AM- and FM-KPFM on 10 nm diameter carbon nanotubes spray-coated on ITO-substrates. 
Both AM- and FM-KPFM were conducted to demonstrate the enhancement in lateral resolution of the GST and amorphous carbon coated AFM tips. We used 5 different Pt tips, 5 GST coated tips and 10 carbon coated tips. Figures 3 (a-c) and 3 (d-f) are representative topography and CPD data collected with platinum tips in AM- and FM-KPFM respectively. In 2-pass AM- and FM-KPFM, the full-width half-maximum (FWHM) of the CPD line cut of a carbon nanotube in Fig. 3 (b) and Fig. 3 (e) with a platinum tip is 82.3 $\mathrm{nm}$ and $68.5 \mathrm{~nm}$ respectively. In AM-KPFM, although the topography is clearly observed, there is little contrast in the potential map. In FM-KPFM, the lateral resolution compared to AM-KPFM is drastically improved. This is because in FM-KPFM, the feedback loop is based on the force gradient. By using the force gradient instead of the force as the feedback mechanism, long-range electrostatic interactions of the cantilever and tip cone can be reduced. Thus, the tip-sample electrostatic interactions measured is mainly contributed by the tip-substrate interaction (i.e. more tip-sensitive technique) ${ }^{28}$. This is particularly useful in the case of our high-resolution probe as the tip modification is localised at the tip. We observed that in both AM- and FM-KPFM with the platinum tip, the FWHM of the CPD signal is greater than the FWHM of the topography signal. In AMKPFM with the platinum tip, the CPD FWHM is 1.70 times that of the topography FWHM. In FM-KPFM, the FWHM of the CPD is 1.37 times that of the topography. We then conducted the same experiments with the switched GST and reconfigured carbon coated tips. Figures 3 (g-l) and (m-r) summarises the KPFM results of switched GST and carbon tips respectively. The resolution obtained in the CPD images are improved in both AM- and FMKPFM compared to when an uncoated platinum tip is used. The FWHM of the CPD in AMKPFM for the GST (carbon) tips improved by 25\% (11\%) from $82.3 \mathrm{~nm}$ to $64.1 \mathrm{~nm}(73.2$ $\mathrm{nm}$ ) and the FWHM in FM-KPFM is improved by 26\% (48\%) from $68.5 \mathrm{~nm}$ to $47.0 \mathrm{~nm}$ (35.8 nm). With the switched GST (carbon) tip, the FWHM of the CPD is 0.80 (1.35) and 0.65 (0.48) times the FWHM of the topography in AM- and FM-KPFM respectively. This results in the ability to resolve below the geometrical limits of the platinum tip. The topographical resolution is similar for both AM- and FM-KPFM. However, it is observed 
that the topographical resolution is consistently slightly better for the platinum tips compared to the carbon tips. To understand why this is the case, we observe the tips in an SEM before and after reconfiguring of the amorphous carbon (Figures 4 (b) and (c)). It can be seen that there is a significant change in shape and volume of the carbon at the apex. The tip radius increases after switching thus resulting in a poorer topographical resolution. No significant change in tip-shape was observed with the switched GST tips. Despite the fact that the tip radius is larger in the carbon tips compared to the Pt tips, the resolution of the CPD images taken with the carbon tips is higher than that of the Pt tips. In Fig. 3 (q), we see that the FWHM of the CPD is even smaller than that of the topography. This is further evidence that carbon filamentation results in enhanced resolution of electrical AFM measurements. We observe that the CPD values differ between the platinum, GST and carbon tips. This is due to the differences in the work functions of the coating material at the apex. Moreover, the different phases of GST and carbon at the apex also affects the CPD measured. Therefore, these results should only be taken in a qualitative manner. Quantitative results may be analysed using a similar method suggested by Keith et al. and is beyond the scope of this paper ${ }^{15}$.

Filamentary Probes for Nanoparticle Manipulation

Another application of the high-resolution electrical probe is in the manipulation of nano objects. One example is the ability to pick nanoparticles with high precision. High precision is rendered if the nanoparticles are picked only at the tip apex. We achieve this by combining our filamentary probes with the technique of dielectrophoresis. Particles, including those which are dielectric will experience a dielectrophoretic force when subjected to a non-uniformed electric field ${ }^{29}$. By exploiting the sharp geometry of AFM tips, Leiterer et al. have used dielectrophoresis to attach silver nanoparticles to AFM tips for tip enhanced Raman spectroscopy (TERS) ${ }^{30}$. However, regardless of the tip size used, nanoparticles on such probes adhere as clusters. This is due to the stray electric field that exists at the sidewalls. Single nanoparticle tips are the preferred geometry for a TERS probe as it excludes complex interactions between the many-nanoparticles 
systems. By using our high electrical resolution tip, we now demonstrate the ability to pick up single nanoparticles. Figure 4 (a) shows a schematic of the process of creating a nanoscale conductive filament and subsequently capturing a single nanoparticle. Secondary electron microscope images were taken at different stages of the dielectrophoresis process. Figure 4 (b) shows the actual SEM image of a carbon coated tip before switching, 4 (c) after switching and 4 (d) after dielectrophoresis to pick up the nanoparticle. We proceed to compare probes in which nanoscale conductive filamentation have been created during switching and probes without this filamentation. Figure 5 (a) shows a representative image of a reconfigured carbon tip after dielectrophoresis. A single nanoparticle is observed at the tip apex and no nanoparticles were observed along the tip cone. We show a large portion of the tip cone to emphasise the locality of using a filamentary probe due to confinement of electric fields. Figure 5 (b) shows a carbon coated tip but without reconfiguration, hence no conductive filaments were formed. After dielectrophoresis, clusters of nanoparticles were observed along the tip. A similar observation of clusters of nanoparticles was made with a normal platinum tip (Fig. 5(c)). This is similar to that observed by Leiterer et al. when they used highly doped silicon tips to pick up silver nanoparticles ${ }^{30}$. In comparison, Figures 5 (d) and (e) show the filamentary GST and carbon probes respectively. We note that there appears to be two nanoparticles attached to the tip in Fig. 5 (d). However, from the transmission electron microscope (TEM) images we see a large improvement in particle trapping resolution with switched GST and carbon tips compared to a tip that has not undergone phase switching (Fig. 5 (b)). We have achieved single nanoparticle resolution in the case of filamentary carbon-coated tips (Fig. 5 (e)). This provides further evidence of the formation of conductive filaments at the apex. We are able to consistently create the conductive filaments at the apex instead of along the side walls because the tip is switched via contact mode conductive AFM where only the apex comes into contact with the substrate. 


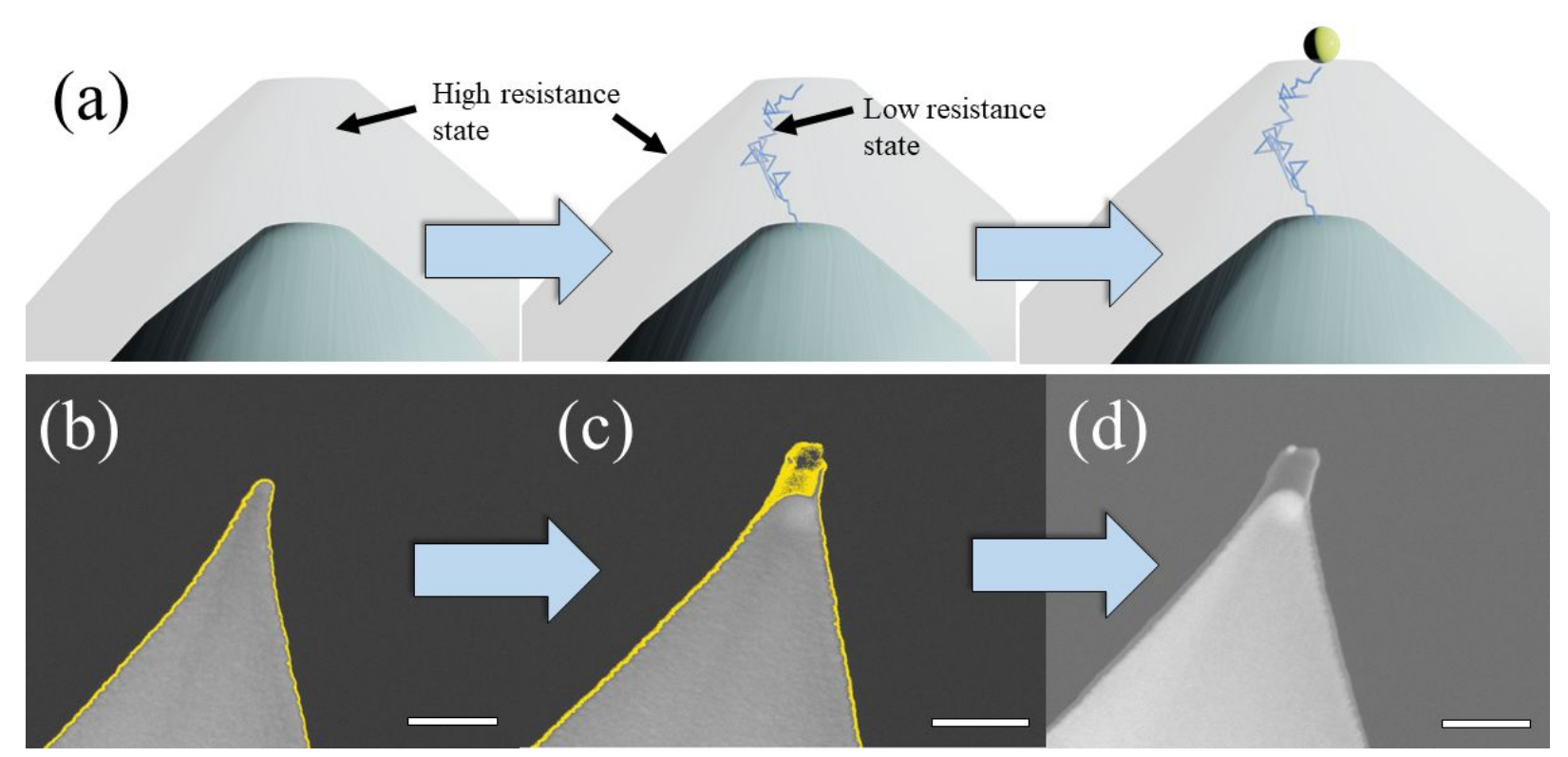

Figure 4: (a) Schematic showing the process of fabricating high-resolution probes. The reconfigurable material is coated onto an AFM tip followed by switching a nanoscale conductive filament at the apex of the tip. The tip can then be used to pick up a nanoparticle through dielectrophoresis or to conduct high-resolution electrical measurements. (b) SEM images of a representative carbon coated platinum tip before threshold switching (c) after switching and (d) after dielectrophoresis to pick up the $20 \mathrm{~nm}$ gold nanoparticle. The yellow colour highlights the carbon coating. The scale bars are $200 \mathrm{~nm}$. 


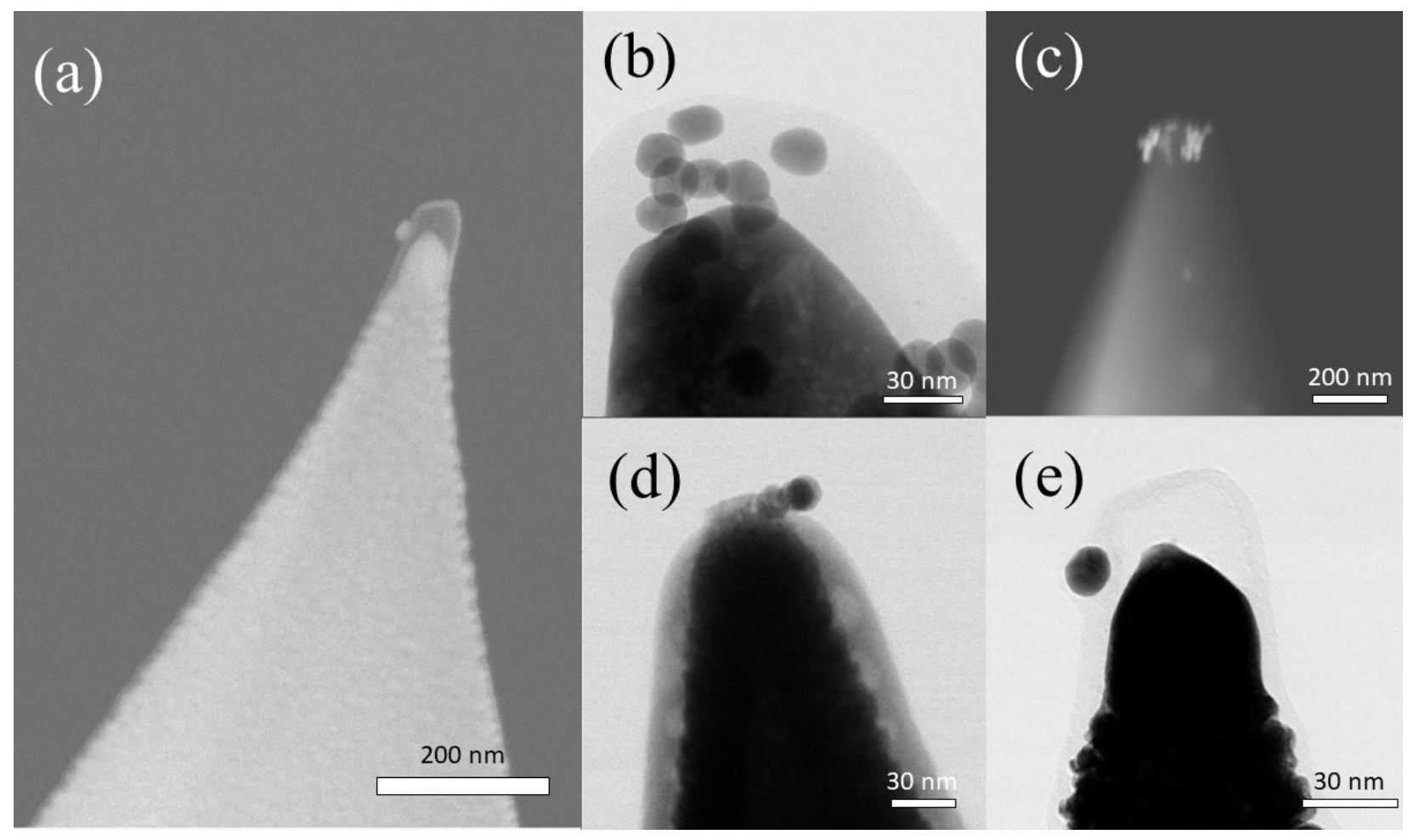

Figure 5: The above images compares the effectiveness of probes for capturing nanoparticles using electric fields with and without conductive filamentation. (a) An SEM (back scattered electron) image of a representative carbon coated platinum tip after dielectrophoresis to pick up a single gold nanoparticle. A large portion of the tip cone illustrates the locality of the electric field resulting in single nanoparticle capturing only at the apex. (b) Carbon coated tip without creation of a nanoscale conductive filament, (c) Pt tip without a coating, (d) filamentary GST tip and (e) filamentary carbon coated tip picks a single Au nanoparticle. Images are a compilation collected by SEM and TEM, thus the contrast reversal.

We note that a previous approach of fabricating electrically high-resolution tips via filamentation has been reported by Hong et al. ${ }^{22}$, where a conventional conductive AFM tip was coated with alumina followed by contacting a bottom metal (palladium) to form a local metal-insulator-metal junction. A conductive pathway is formed through the alumina due to metal migration from the substrate. Our approach of coating with a reconfigurable material followed by switching offers several advantages. The phase change is intrinsic to the coating and is independent of the substrate or tip material as 
in the case with metallic filamentation described by Hong et al. This means that reconfiguration and measurement can be done in-situ. Moreover, the use of carbon as the active coating material offers the possibilities of functionalisation as well as being widely available and readily coated onto AFM tips.

\section{Conclusion}

In summary, we have demonstrated a readily available method for manufacturing highresolution electrical AFM probes with nanoscale precision not attainable by standard probes using controlled phase switching of GST and amorphous carbon at the nanoscale. Virtually any probe can be turned into a high-resolution probe in-situ with this method. This will benefit all electrical AFM techniques. Moreover, as sputter deposition is used, mass production of GST probes can be achieved. We confirmed the presence of conductive filamentation by observing IV curves during switching and by successful implementation of two applications. We demonstrated high-resolution AM- and FMKPFM on carbon nanotube samples and the ability to pick up a single nanoparticle at the apex by dielectrophoresis at ambient conditions opening the door to a new method for pick and place assembly at the nanoscale.

Materials and methods

Tip preparation and characterisation

GST is sputtered on the platinum coated tips with chromium as an adhesion layer while amorphous carbon is deposited by electron beam-induced deposition (EBID). GST is sputtered with a Nordiko sputtering tool at $30 \mathrm{~W}, 5 \mathrm{mT}$ Trr and $15 \mathrm{sccm}$ of Argon pressure and flow respectively. EBID is performed in a ZEISS Merlin SEM with an electron beam of $30 \mathrm{kV}$ and $500 \mathrm{pA}$ focused at the tip apex. Carbon deposition thickness 
is monitored in-situ. The use of EBID in a scanning electron microscope (SEM) allows high spatial accuracy in the deposition of amorphous carbon at the apex of the AFM tips. The GST and amorphous carbon at the apex of the AFM tip are switched from a HRS to a LRS in conductive AFM by ramping up an external voltage between the probe and the sample (Fig. 2(a)). The modified tip is lowered into contact with a conducting substrate. A $510 \mathrm{k} \Omega$ resistor is placed in series to limit the current flow through the tip when the LRS is achieved. This protects the tip from high currents, which could melt and damage the coating. The voltage is ramped up until a sudden spike in current is observed.

\section{Substrate preparation}

Ethylene-vinyl acetate (EVA) and multi-walled carbon nanotube (MWCNT) solution preparation were carried out as described by Mazzotta et al ${ }^{31}$. The solution was deposited on ITO substrates (TFD Inc., USA) with a custom-made open science hardware spray coater [reference to http://afmd.github.io/LANDS].

\section{KPFM Characterisation}

KPFM measurements were carried out on an Asylum Research MFP3D AFM. A variety of probes were used as is or coated with GST or carbon. These include platinum coated (TAP150E-G), silicon (TAP300-G) and diamond (AIO-DD) coated probes. The typical tip radius is $25 \mathrm{~nm}$. The topography feedback was performed in AM at the resonance frequency of the cantilever and the oscillation amplitude was kept to about $40 \mathrm{~nm}$. To perform FM-KPFM, we used an external lock-in amplifier (Zurich Instruments, HF2LI). The FM-KPFM method used is the FM sideband method as described by Axt et al ${ }^{32}$. We electrically excite the cantilever at $\omega_{\mathrm{e}}=2.5 \mathrm{kHz}$ with a driving voltage of $3 \mathrm{~V}$ and demodulate at the sidebands $\omega_{\mathrm{m}} \pm \omega_{\mathrm{e}}$, where $\omega_{\mathrm{m}}$ is the frequency of mechanical oscillations. For the dual-pass AM-KPFM, the topography and CPD measurements are completely decoupled. In the first pass, the topographic information is captured in tapping mode. During the second pass, the mechanical excitation of the cantilever is turned off and the tip follows the topography as imaged in the first pass at a defined lift height of $20 \mathrm{~nm}$ 
above the surface while measuring the CPD. All scans were conducted at $0.5 \mathrm{~Hz}$ and repeated multiple times.

\section{TEM Characterisation}

Transmission electron microscopy was carried out in TEM and Scanning TEM modes in conjunction with EDX spectroscopy maps for elemental confirmation and distribution. A JEOL GrandARM was used at $300 \mathrm{kV}$ with an Oxford Instruments XMAX 100 EDX detector. The AFM tip devices were held into the optimum z-height of the objective lens with a custom-fabricated clamping tip compatible with the JEOL quickexchange holder ${ }^{33}$.

Dielectrophoresis to capture single nanoparticle

We used the phase change material coated probes with a nanoscale conductive filament as one electrode and a planar platinum substrate as the other electrode. A voltage of 10 $\mathrm{V}$ peak-to-peak at $100 \mathrm{kHz}$ was applied for $60 \mathrm{~s}$. The distance between the AFM probe and substrate was set to $500 \mathrm{~nm}$.

\section{Contributions}

E.J.H.S and H.B conceived the concept of coating probes with reconfigurable material. S.G.S conceived the concept for high-resolution Kelvin probe force microscopy. E.J.H.S and B.F.P conceived the concept of nano-particles pickup using dielectrophoresis. G.M prepared the nanotubes. E.J.H.S conducted 
all experiments, with the assistance of J.S.K, B.F.P and S.G.S. All authors contributed to the manuscript.

\section{Acknowledgements}

We thank Professor Stefan Weber for helpful discussions. E.J.H.S acknowledges an A*STAR Scholarship, and S.G.S acknowledges a Felix Scholarship that supports their research. G.M. acknowledges EPSRC for the funding through the Centre for Doctoral Training in New and Sustainable PV (EP/L01551X/1) and University College, Oxford, for the Oxford-Radcliffe scholarship. This work was primarily supported by EPSRC, European Union's Horizon 2020 programme via the FunComp project and the John Fell Fund. We thank Diamond Light Source for access and support in use of the electron Physical Science Imaging Centre (E02, mg22207) that contributed to the results presented. The authors declare no competing financial interest.

Notes

H.B. holds shares and serves as a company board director at Bodle Technologies Ltd. H.B. is also an employee of the University of Oxford. The authors declare no competing financial interest.

Supporting Information Available:

Further SEM images of a tip after scanning, CNT characterisations, STEM and EDX elemental maps of carbon and GST tips and additional KPFM data.

References

(1) Filatov, D.; Kazantseva, I.; Antonov, D.; Antonov, I.; Shenina, M.; Pavlov, D.; Gorshkov, O. Conductive Atomic Force Microscopy Study of the Resistive Switching in YttriaStabilized Zirconia Films with Au Nanoparticles. Scanning 2018, 2018.

(2) Bürgi, L.; Richards, T. J.; Friend, R. H.; Sirringhaus, H. Close Look at Charge Carrier Injection in Polymer Field-Effect Transistors. J. Appl. Phys. 2003, 94 (9), 6129-6137. 
(3) Ji, Y.; Pan, C.; Zhang, M.; Long, S.; Lian, X.; Miao, F.; Hui, F.; Shi, Y.; Larcher, L.; Wu, E.; et al. Boron Nitride as Two Dimensional Dielectric: Reliability and Dielectric Breakdown. Appl. Phys. Lett. 2016, 108 (1), 12905.

(4) Wang, Z. L.; Song, J. Piezoelectric Nanogenerators Based on Zinc Oxide Nanowire Arrays. Science (80-. ). 2006, 312 (5771), 242-246.

(5) Zhou, C.; Kong, J.; Dai, H. Electrical Measurements of Individual Semiconducting Single-Walled Carbon Nanotubes of Various Diameters. Appl. Phys. Lett. 2000, 76 (12), 1597-1599.

(6) Cui, X. D.; Primak, A.; Zarate, X.; Tomfohr, J.; Sankey, O. F.; Moore, A. L.; Moore, T. A.; Gust, D.; Harris, G.; Lindsay, S. M. Reproducible Measurement of Single-Molecule Conductivity. Science (80-. ). 2001, 294 (5542), 571-574.

(7) Shi, Y.; Ji, Y.; Hui, F.; Nafria, M.; Porti, M.; Bersuker, G.; Lanza, M. In Situ Demonstration of the Link Between Mechanical Strength and Resistive Switching in Resistive Random-Access Memories. Adv. Electron. Mater. 2015, 1 (4), 1400058.

(8) Leblebici, S. Y.; Leppert, L.; Li, Y.; Reyes-Lillo, S. E.; Wickenburg, S.; Wong, E.; Lee, J.; Melli, M.; Ziegler, D.; Angell, D. K.; et al. Facet-Dependent Photovoltaic Efficiency Variations in Single Grains of Hybrid Halide Perovskite. Nat. Energy 2016, 1 (8), 16093.

(9) Murrell, M. P.; Welland, M. E.; O’Shea, S. J.; Wong, T. M. H.; Barnes, J. R.; McKinnon, A. W.; Heyns, M.; Verhaverbeke, S. Spatially Resolved Electrical Measurements of SiO2 
Gate Oxides Using Atomic Force Microscopy. Appl. Phys. Lett. 1993, 62 (7), 786-788.

(10) Shi, Y.; Ji, Y.; Hui, F.; Iglesias, V.; Porti, M.; Nafria, M.; Miranda, E.; Bersuker, G.; Lanza, M. Elucidating the Origin of Resistive Switching in Ultrathin Hafnium Oxides through High Spatial Resolution Tools. ECS Trans. 2014, 64 (14), 19-28.

(11) Nellist, M. R.; Laskowski, F. A. L.; Qiu, J.; Hajibabaei, H.; Sivula, K.; Hamann, T. W.; Boettcher, S. W. Potential-Sensing Electrochemical Atomic Force Microscopy for in Operando Analysis of Water-Splitting Catalysts and Interfaces. Nat. Energy 2018, 3 (1), 46.

(12) Shiota, T.; Nakayama, K. Atom-Resolved Imaging of the Potential Distribution at Si (111) 7 x 7 Surfaces. Jpn. J. Appl. Phys. 2002, 41 (10B), L1178.

(13) Bhaskaran, H.; Sebastian, A.; Drechsler, U.; Despont, M. Encapsulated Tips for Reliable Nanoscale Conduction in Scanning Probe Technologies. Nanotechnology 2009, 20 (10), 105701.

(14) Sadewasser, S.; Villanueva, G.; Plaza, J. A. Special Cantilever Geometry for the Access of Higher Oscillation Modes in Atomic Force Microscopy. Appl. Phys. Lett. 2006, 89 (3), 33106.

(15) Brown, K. A.; Satzinger, K. J.; Westervelt, R. M. High Spatial Resolution Kelvin Probe Force Microscopy with Coaxial Probes. Nanotechnology 2012, 23 (11), 115703.

(16) Hafner, J. H.; Cheung, C. L.; Lieber, C. M. Growth of Nanotubes for Probe Microscopy 
Tips. Nature 1999, 398 (6730), 761.

(17) Zhao, M.; Sharma, V.; Wei, H.; Birge, R. R.; Stuart, J. A.; Papadimitrakopoulos, F.; Huey, B. D. Ultrasharp and High Aspect Ratio Carbon Nanotube Atomic Force Microscopy Probes for Enhanced Surface Potential Imaging. Nanotechnology 2008, 19 (23), 235704.

(18) Wilson, N. R.; Macpherson, J. V. Carbon Nanotube Tips for Atomic Force Microscopy. Nat. Nanotechnol. 2009, 4 (8), 483.

(19) Wilson, N. R.; Cobden, D. H.; Macpherson, J. V. Single-Wall Carbon Nanotube Conducting Probe Tips. J. Phys. Chem. B 2002, 106 (51), 13102-13105.

(20) Yang, F.; Xu, L.; Chen, J.; Xu, J.; Yu, Y.; Ma, Z.; Chen, K. Nanoscale Multilevel Switching in Ge2Sb2Te5 Thin Film with Conductive Atomic Force Microscopy. Nanotechnology 2015, 27 (3), 35706.

(21) Sebastian, A.; Pauza, A.; Rossel, C.; Shelby, R. M.; Rodr'\iguez, A. F.; Pozidis, H.; Eleftheriou, E. Resistance Switching at the Nanometre Scale in Amorphous Carbon. New J. Phys. 2011, 13 (1), 13020.

(22) Hong, S. S.; Cha, J. J.; Cui, Y. One Nanometer Resolution Electrical Probe via Atomic Metal Filament Formation. Nano Lett. 2011, 11 (1), 231-235. https://doi.org/10.1021/nl103603v.

(23) Hoppe, H.; Glatzel, T.; Niggemann, M.; Hinsch, A.; Lux-Steiner, M. C.; Sariciftci, N. S. 
Kelvin Probe Force Microscopy Study on Conjugated Polymer/fullerene Bulk Heterojunction Organic Solar Cells. Nano Lett. 2005, 5 (2), 269-274.

(24) Liu, L.; Li, G. Electrical Characterization of Single-Walled Carbon Nanotubes in Organic Solar Cells by Kelvin Probe Force Microscopy. Appl. Phys. Lett. 2010, 96 (8), 33.

(25) Sarwat, S. G.; Tweedie, M.; Porter, B. F.; Zhou, Y.; Sheng, Y.; Mol, J.; Warner, J.; Bhaskaran, H. Revealing Strain-Induced Effects in Ultrathin Heterostructures at the Nanoscale. Nano Lett. 2018, 18 (4), 2467-2474.

(26) Clack, N. G.; Salaita, K.; Groves, J. T. Electrostatic Readout of DNA Microarrays with Charged Microspheres. Nat. Biotechnol. 2008, 26 (7), 825.

(27) Finot, E.; Leonenko, Y.; Moores, B.; Eng, L.; Amrein, M.; Leonenko, Z. Effect of Cholesterol on Electrostatics in Lipid- Protein Films of a Pulmonary Surfactant. Langmuir 2010, 26 (3), 1929-1935.

(28) Zerweck, U.; Loppacher, C.; Otto, T.; Grafström, S.; Eng, L. M. Accuracy and Resolution Limits of Kelvin Probe Force Microscopy. Phys. Rev. B 2005, 71 (12), 125424.

(29) Pohl, H. A. Dielectrophoresis. Behav. neutral matter nonuniform Electr. fields 1978.

(30) Leiterer, C.; Wünsche, E.; Singh, P.; Albert, J.; Köhler, J. M.; Deckert, V.; Fritzsche, W. High Precision Attachment of Silver Nanoparticles on AFM Tips by Dielectrophoresis. Anal. Bioanal. Chem. 2016, 408 (13), 3625-3631. https://doi.org/10.1007/s00216- 
016-9447-6.

(31) Mazzotta, G.; Dollmann, M.; Habisreutinger, S. N.; Christoforo, M. G.; Wang, Z.; Snaith, H. J.; Riede, M. K.; Nicholas, R. J. Solubilization of Carbon Nanotubes with EthyleneVinyl Acetate for Solution-Processed Conductive Films and Charge Extraction Layers in Perovskite Solar Cells. ACS Appl. Mater. Interfaces 2018, 11 (1), 1185-1191.

(32) Axt, A.; Hermes, I. M.; Bergmann, V. W.; Tausendpfund, N.; Weber, S. A. L. Know Your Full Potential : Quantitative Kelvin Probe Force Microscopy on Nanoscale Electrical Devices. 2018, 1809-1819. https://doi.org/10.3762/bjnano.9.172.

(33) Pacios, M.; Hosseini, P.; Fan, Y.; He, Z.; Krause, O.; Hutchison, J.; Warner, J. H.; Bhaskaran, H. Direct Manufacturing of Ultrathin Graphite on Three-Dimensional Nanoscale Features. Sci. Rep. 2016, 6 (March), 1-8. https://doi.org/10.1038/srep22700. 
56

57

58

59

Filmentary high resolution probe concept confines electric fields to a nanoscale region $70 \times 34 \mathrm{~mm}(96 \times 96 \mathrm{DPI})$ 